Europhys. Lett., 64 (1), pp. 131-136 (2003)

\title{
Impact of Poisson synaptic inputs with a changing rate on weak-signal processing
}

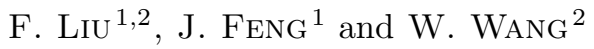 \\ 1 School of Cognitive and Computing Sciences, University of Sussex \\ Brighton, BN1 9QH, UK \\ 2 Department of Physics, Nanjing University - Nanjing 210093, PRC
}

(received 14 March 2003; accepted in final form 25 July 2003)

PACS. 87.16.Ac - Theory and modeling; computer simulation.

PACS. 05.40.Ca - Noise.

\begin{abstract}
We explore the response of a single Hodgkin-Huxley neuron to a local field potential input, which is modeled as a subthreshold harmonic signal, plus synaptic inputs from independent Poisson processes. The synaptic input rate is periodically modulated with the same frequency as the signal. The neural behavior depends remarkably on the signal frequency. The neuron is more sensitive to the signals with frequencies ranging from 30 to $65 \mathrm{~Hz}$ and can produce regular output spikes with a low variability. Increasing the mean synaptic input rate can improve the detection of the subthreshold signal. These results further verify that noise can play a constructive role in signal processing of neurons.
\end{abstract}

Stochastic resonance (SR) has attracted considerable attention since it was shown that the response of nonlinear systems to weak signals can be optimized by noise [1]. Although the role of SR in sensory neuronal systems was reported extensively [1-4], its role in the central nervous system has been investigated only recently [5-7]. The synaptic connectivity of the neocortex is very dense; for example, each pyramidal cell receives 5000-60000 synaptic inputs [8], which can act as a potential noise source. Thus, it is of interest to explore the impact of such endogenous noise, rather than the combined effect of all noisy inputs such as Gaussian noise [5], on weak-signal processing of cortical neurons. It was demonstrated that synaptic noise indeed improves the detection of subthreshold synaptic signals in hippocampal CA1 neurons [6]. Neocortical pyramidal neurons can also exhibit SR-like behavior when subject to subthreshold periodic stimuli plus synaptic noise [7].

It is well known that a Poisson process may constitute a first-order approximation description of cortical spike trains [9]. A large body of literature has studied neuronal response to homogeneous Poisson synaptic input (see refs. [10,11] and references therein). In contrast to refs. $[6,7]$, in this letter a neuron is assumed to receive synaptic inputs from inhomogeneous Poisson processes, whose changing rate represents the temporal information. Such an assumption is of biological relevance; for instance, when an MT neuron in a behaving monkey responds to patterns of randomly moving dots, its output spike trains can be characterized by a Poisson process with a time-varying rate (cf. fig. 15.11 in [9]).

(c) EDP Sciences 
Motivated by the above considerations, here we explore the response of a single neuron to a subthreshold (local field potential) signal $s(t)$ plus synaptic noise $I_{\text {noise }}(t)$. We shall show that the neuron is more sensitive to the signals with frequencies ranging from 30 to $65 \mathrm{~Hz}$ and can generate regular output spikes with a low variability. In the frequency sensitivity range, increasing the mean synaptic input rate (noise intensity) can definitely enhance the signal detection. Outside that the neuron exhibits more or less the SR-like behavior.

The neural dynamics is described by the Hodgkin-Huxley (HH) equations

$$
\begin{aligned}
C_{m} \frac{\mathrm{d} V}{\mathrm{~d} t}= & -g_{N_{a}} m^{3} h\left(V-V_{N_{a}}\right)-g_{K} n^{4}\left(V-V_{K}\right)- \\
& -g_{l}\left(V-V_{l}\right)+s(t)+I_{\text {noise }}(t), \\
\frac{\mathrm{d} x}{\mathrm{~d} t}= & \alpha_{x}(V)(1-x)-\beta_{x}(V) x, \quad x=m, h, n .
\end{aligned}
$$

Here the typical values of parameters and forms of functions are taken, just the same as used in refs. [12,13]. That is, $C_{m}=1 \mu \mathrm{F} / \mathrm{cm}^{2}, V_{N_{a}}=50 \mathrm{mV}, V_{K}=-77 \mathrm{mV}, V_{l}=-54.4 \mathrm{mV}, g_{N_{a}}=$ $120 \mathrm{mS} / \mathrm{cm}^{2}, g_{K}=36 \mathrm{mS} / \mathrm{cm}^{2}, g_{l}=0.3 \mathrm{mS} / \mathrm{cm}^{2}$, and $\alpha_{m}=0.1(V+40) /\left(1-e^{-(V+40) / 10}\right)$, $\beta_{m}=4 e^{-(V+65) / 18}, \alpha_{h}=0.07 e^{-(V+65) / 20}, \beta_{h}=1 /\left(1+e^{-(V+35) / 10}\right), \alpha_{n}=0.01(V+55) /(1-$ $\left.e^{-(V+55) / 10}\right)$, and $\beta_{n}=0.125 e^{-(V+65) / 80}$. All the currents are in units of $\mu \mathrm{A} / \mathrm{cm}^{2} . s(t)$ is a subthreshold signal, $\cos \left(2 \pi f_{s} t\right)$, corresponding to the input generated by the local field potential. $I_{\text {noise }}$ represents the sum of excitatory and inhibitory synaptic inputs and is defined as

$$
I_{\text {noise }}(t)=-\frac{J}{N} \sum_{i=1}^{N} \sum_{\left\{t_{i}^{k}\right\}} \alpha\left(t-t_{i}^{k}\right)\left(V(t)-E_{i}\right)
$$

with $\alpha\left(t-t_{i}^{k}\right) \equiv \alpha\left(t^{\prime}\right)=\frac{t^{\prime}}{\tau} e^{-\frac{t^{\prime}}{\tau}}$ for $t^{\prime}>0$ and otherwise $\alpha\left(t^{\prime}\right)=0 . \quad \tau$ is taken as $2 \mathrm{~ms}$. $\left\{t_{i}^{k}\right\}$ is Poisson distributed with a time-varying rate $r_{i}(t)$, but is modified by inclusion of an absolute refractory period, which is Gaussian distributed with $5 \mathrm{~ms}$ mean and $2 \mathrm{~ms}$ standard deviation $[9,14]$. As a first approximation, we represent $r_{i}(t)$ by its time average and Fourier transform at the signal frequency $f_{s}$, i.e. $r_{i}(t)=\mu_{i}\left(1+\lambda_{i} \cos \left(2 \pi f_{s} t\right)\right)$ (for simplicity, $\lambda_{i}$ is set to 1 throughout). Note that a similar assumption was made in $[15,16] . E_{i}$ is taken as -80 or $0 \mathrm{mV}$ for inhibitory and excitatory synapses, respectively. $J$ is the peak conductance and is set to $2 \mathrm{mS} / \mathrm{cm}^{2}$. Since pyramidal cells are generally four times as numerous as interneurons in the neocortex [8], here the fraction of synapses which are excitatory is assumed to be 0.8 . The number of synapses is taken as $N=100$, unless specified elsewhere.

Numerical integration is performed by the fourth-order Runge-Kutta algorithm and the time step is $500 / 32768 \mathrm{~ms}$. The neural behavior is characterized by the mean output firing rate $R$, the coefficient of variation $\left(C_{v}\right)$ of output interspike intervals (ISIs), and the output signal-to-noise ratio (SNR). $R$ is evaluated as the spike-count rate over the duration of $10 \mathrm{~s}$ after the transients are discarded, while $C_{v}$ is defined as the ratio of the standard deviation to the mean of ISI. To compute power spectral density (PSD), the time course of $V(t)$ is first converted into a sequence of standard pulses $U(t)$ with $U_{H}=1$ of width $2 \mathrm{~ms}$ and $U_{L}=0$ corresponding to the firing and non-firing states, respectively (the firing threshold is taken as $-20 \mathrm{mV})$. The SNR is defined as $10 \log _{10}(\Gamma / B)$ with $\Gamma$ and $B$ representing the height of the signal peak and the mean amplitude of background noise at the signal frequency $f_{s}$ in the PSD, respectively. All reported results are taken on an average over 100 different realizations.

We first discuss how the neural responses vary with the signal frequency when the mean input rate $\mu_{i}$ is uniformly distributed between 10 and $60 \mathrm{~Hz}$. Since the input rate is periodically 


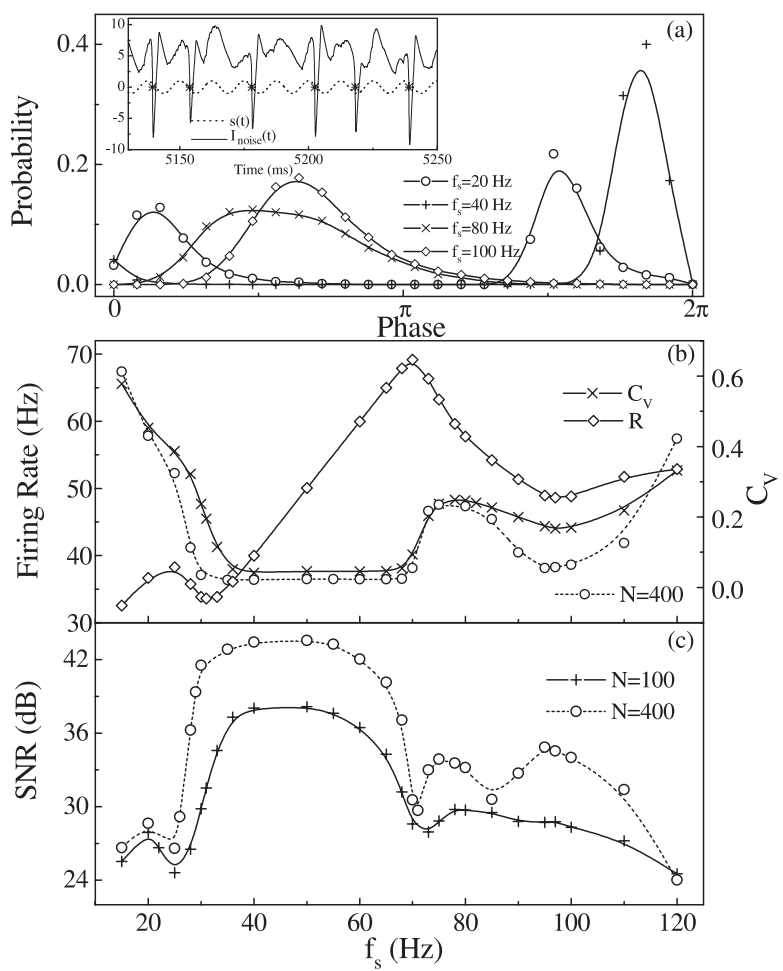

Fig. 1 - Frequency sensitivity in response to a weak periodic signal plus synaptic noise. The mean input rate $\mu_{i}$ is uniformly distributed between 10 and $60 \mathrm{~Hz}$. (a) The firing phase histograms, i.e. the probability of an output spike being produced at a particular phase of the periodic signal, for $f_{s}=20,40,80$ and $100 \mathrm{~Hz}$, respectively. The inset shows the time courses of $s(t)$ and $I_{\text {noise }}(t)$ for $f_{s}=80 \mathrm{~Hz}$. The mark ${ }^{*}$ indicates the timing of output spikes. (b) $R$ and $C_{v}$ and (c) the SNR vs. the signal frequency. The dashed curves are for $N=400$.

modulated, input spikes are more likely to arrive at synapses when the signal takes a positive value. As a result, the neural firings can be coherent with the (local field potential) signal, but the degree of phase locking depends remarkably on the signal frequency (fig. 1a). For $f_{s}=40 \mathrm{~Hz}$ most spikes are discharged at $2 \sim 4 \mathrm{~ms}$ prior to the peaks of the stimulus, whereas for $f_{s}=80$ or $100 \mathrm{~Hz}$ the firing phase is widely distributed in the first half-period.

For $f_{s} \leq 35 \mathrm{~Hz}$ the firing rate $R$ is larger than $f_{s}$, and their difference decreases with increasing $f_{s}$ (fig. 1b). As $f_{s}$ rises, $R$ is equal to $f_{s}$ up to $f_{s}=69 \mathrm{~Hz}$ and then declines monotonically until $f_{s}=97 \mathrm{~Hz}$. For $f_{s}>100 \mathrm{~Hz} R$ increases with $f_{s}$ but is always smaller than $f_{s} / 2$. Accordingly, there also exist four frequency ranges in the curve of $C_{v} v s . f_{s}$ (fig. 1b). Especially, $C_{v}$ remains nearly the same value of 0.04 when $40 \leq f_{s} \leq 68 \mathrm{~Hz}$ and is always below 0.3 for $30 \leq f_{s}<120 \mathrm{~Hz}$. This means that the neuron can generate regular output spikes even in the presence of noise. The present result is different from that in ref. [15], wherein $C_{v}$ is close to 1 when the leaky integrate-and-fire (IF) model neuron is subject to the balanced synaptic inputs. In fact, the $\mathrm{HH}$ model is classified as type-II excitability in terms of the relationship between firing rate and constant stimulus, whereas the IF model is classified as type I. It was argued that type-II neurons can produce output spikes with $C_{v}<0.3$ in response to stochastic synaptic input [17]. Furthermore, it was demonstrated that the $\mathrm{HH}$ 

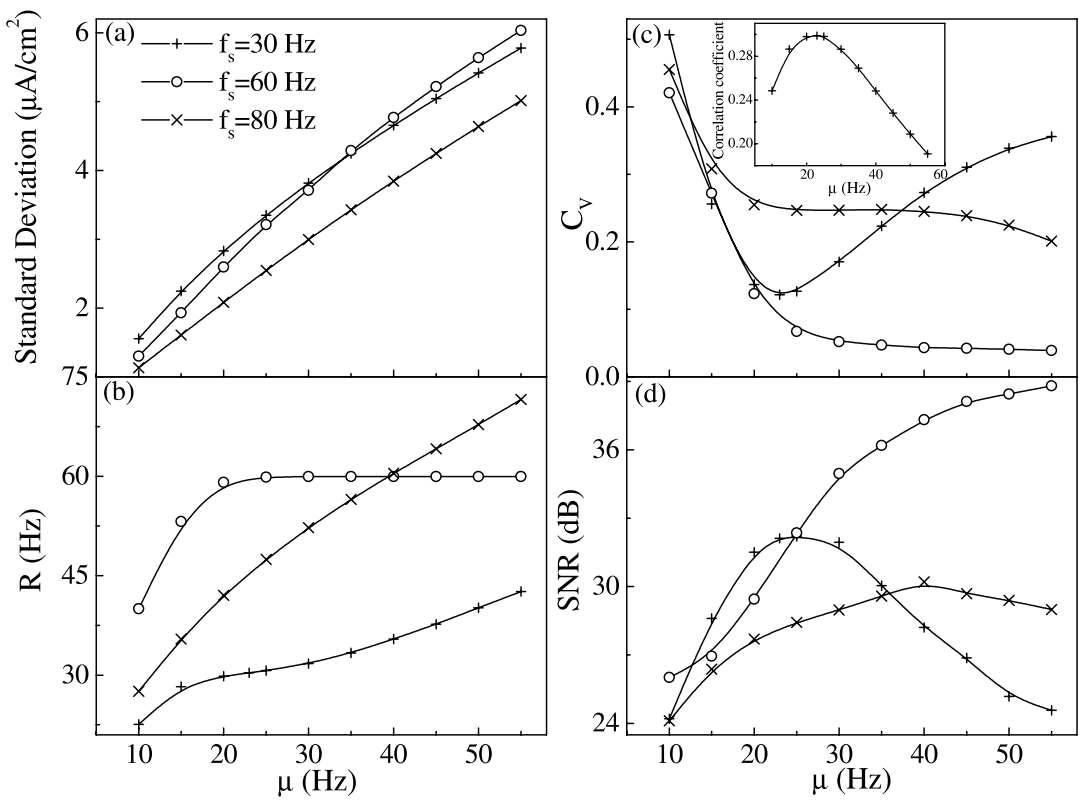

Fig. 2 - Synaptic noise can enhance the neuronal response. Here each synaptic input is of an identical mean rate $\mu$. (a) The standard deviation of $I_{\text {noise }}(t)$, (b) $R$, (c) $C_{v}$ and (d) the SNR vs. $\mu$ for $f_{s}=30$ $(+), 60(\circ)$ and $80 \mathrm{~Hz}(\times)$, respectively. The inset of (c) shows the maximum of the coefficient of correlation between the signal $s(t)$ and the firing pulse $U(t)$ for $f_{s}=30 \mathrm{~Hz}$.

and IF models behave in totally opposite ways when subject to correlated synaptic inputs [13].

Obviously, the SNR is roughly in agreement with $C_{v}$ varying with $f_{s}$ and takes relatively large values for $33 \leq f_{s} \leq 65 \mathrm{~Hz}$ (fig. 1c). We also depict $C_{v}$ and the SNR for the case of $N=400$ to investigate the influence of the number of synapses on neuronal behavior. Generally speaking, the SNR becomes larger while $C_{v}$ decreases with increasing $N$ (of course they will saturate at large $N$ ). But their general tendency of varying with $f_{s}$ changes little (nevertheless, the local maximum around $f_{s}=95 \mathrm{~Hz}$ becomes more pronounced under $N=$ 400 since the neural firing exhibits a coherent behavior with $R=f_{s} / 2$ ). The SNR also takes large values for $30 \leq f_{s} \leq 65 \mathrm{~Hz}$. These results indicate that the neuron is more sensitive to the signals with frequencies in the range of $30 \sim 65 \mathrm{~Hz}$, capable of reliably retaining and transmitting the temporal information contained in the stimulus. Such frequency sensitivity results from the resonance effect between the subthreshold membrane potential oscillation and the periodic signal. It was shown that a noise-free $\mathrm{HH}$ neuron exhibits a damped oscillation, whose frequency is around $60 \mathrm{~Hz}$ for $I=0$, in response to a transient synaptic input [18]. As a result, resonance improves the ability of neurons to respond selectively to inputs at preferred frequencies, which may be of functional significance. Similar frequency sensitivity in weak-signal detection was reported experimentally [3].

Now let us assume that each synaptic input has an identical rate $\mu_{i}=\mu$ but is still independent of any other. We turn to explore how $\mu$ affects neural response under a fixed $f_{s}$. As seen in the inset of fig. 1a, the synaptic current $I_{\text {noise }}(t)$ is closely correlated with $s(t)$ and shows to fluctuate around a mean value except for large excursions corresponding to spike occurrence. The standard deviation of $I_{\text {noise }}$ increases monotonically with $\mu$ (so does the mean of $I_{\text {noise }}$ ) (fig. 2a). Therefore, we can take $\mu$ as a measure of noise intensity. As will 
be demonstrated, the neural behavior as a function of $\mu$ can be roughly classified based on $f_{s}<40 \mathrm{~Hz}, 40 \leq f_{s}<75 \mathrm{~Hz}$ or $f_{s} \geq 75 \mathrm{~Hz}$, as in fig. 1 .

Figure 2b-d plots $R, C_{v}$ and the SNR vs. $\mu$, respectively. For $f_{s}=30 \mathrm{~Hz}$, as more input spikes arrive coincidentally at synapses with increasing $\mu$, the effective synaptic current becomes larger and thus $R$ increases with $\mu$ but varies slowly. Meanwhile, $C_{v}$ first declines to a minimum at $\mu_{0}=23 \mathrm{~Hz}$ and then rises. Note that $R$ is equal to $30 \mathrm{~Hz}$ at $\mu_{0}$. The $\mathrm{SNR}$ also first rises up to a maximum at $\mu_{0}$ and then drops with increasing $\mu$. That is, the maximum SNR is reached when the firings are most correlated with the signal. This can be clearly seen in the inset of fig. 2c. The maximum of the coefficient of correlation between $s(t)$ and $U(t)$ is consistent with the SNR and also takes its maximum at $\mu_{0}$. Therefore, in the presence of synaptic noise the neural behavior can be optimized by suitable noise level. The above conclusion also holds for the case of $f_{s} \geq 75 \mathrm{~Hz}$, but the change of $C_{v}$ and the SNR at large $\mu$ becomes much less remarked.

For $f_{s}=60 \mathrm{~Hz}$, when $\mu$ exceeds $25 \mathrm{~Hz}, R$ equals $f_{s}$ while $C_{v}$ takes a much smaller value and also decreases slightly with $\mu$. Accordingly, the SNR rises monotonically but is nearly saturated at large $\mu$. This is so because at moderate $\mu$ the neuron fires one spike in each driving cycle and the firing correlation with the signal is strengthened with increasing $\mu$.

Moreover, if we fix $\mu$ and vary the synaptic conductance $J$, similar behavior can be observed (data not shown). Therefore, increasing the level of synaptic noise can improve the weaksignal detection, but such an effect is closely related to the signal frequency. For $f_{s}<40$ or $f_{s} \geq 75 \mathrm{~Hz}$ the SNR goes through a maximum as a function of $\mu$ in a manner similar to that in the context of SR [1]. As mentioned above, since the synaptic noise is multiplicative, both its mean and standard variance monotonically increase with $\mu$. It is the balance between these two opposing effects that results in the optimal response. But in the case of additive noise, typical SR results from the cooperative effect between noise-induced firing and the input signal. In contrast, for $40 \leq f_{s}<75 \mathrm{~Hz}$ the SNR first monotonically increases but is saturated at large $\mu$. In any case, the neural behavior can always be optimized by the presence of synaptic noise.

It is worth noting that assuming the rate of Poisson process varies periodically is equivalent to introducing a correlation among synaptic inputs [15], although each input spike train is generated independently. We have also investigated the case wherein the neuron is subject to synaptic inputs from independent homogeneous Poisson processes (data not shown). Compared with that, the neuron here can produce the output spikes with a much lower variability. This is in agreement with the conclusion that for the HH model $C_{v}$ is a decreasing function of input correlation [13]. This may be of biological significance. Since neurons with similar functions always group together and fire in a correlated way, their downstream neurons may thus be able to efficiently process information when subject to these correlated synaptic inputs. Notice that it was also claimed elsewhere $[19,20]$ that at realistic levels of stochastic synaptic inputs neurons can effectively integrate a large number of random inputs to produce an output with a low variability.

In the presence of Poisson synaptic inputs, the neuron is more sensitive to the signals with frequencies ranging from 30 to $65 \mathrm{~Hz}$. This is reminiscent of the $40 \mathrm{~Hz}$ oscillations (with frequencies between 30 and $70 \mathrm{~Hz}$ ) observed in various brain areas [21], which play functional roles such as pattern segmentation and feature binding. The present results imply that such frequency sensitivity can considerably enhance effects of weak $40 \mathrm{~Hz}$ oscillations. This may also give us an enlightenment on why $\gamma$ rhythms are so ubiquitous in information processing and functional realization.

Finally, we make a remark on the input rate $r_{i}=\mu_{i}\left(1+\lambda_{i} \cos \left(2 \pi f_{i} t\right)\right)$ considered in this letter. Here we assume $f_{i}=f_{s}$, the (local field potential) signal frequency, which may be a 
somewhat arbitrary form for modeling synaptic input (see, for example [22], on experimental data). If $f_{i}$ is considered to be distributed within a frequency range, there might appear some new phenomena. This deserves a further study.

In conclusion, we have investigated the response of a single HH neuron to a subthreshold harmonic signal plus synaptic inputs, each following a Poisson process with a periodically varying rate. The results illustrate how the presence of synaptic noise can enhance the neural response and how the neuron can reliably transmit the temporal information about the stimulus by producing regular output spikes with $C_{v}<0.3$. The neuron can attain an optimal response by adjusting the synaptic input, say the mean input rate or the synaptic conductance. These suggest that noise can play a constructive role in information processing of cortical neurons.

$$
* * *
$$

This work is supported by the NNSF of China (grant no. 30070208). JF acknowledges the support from the EPSRC (grant no. R54569), the Royal Society and an exchange grant of the Royal Society.

\section{REFERENCES}

[1] Gammatoni L., Hänggi P., Jung P. and Marchesoni F., Rev. Mod. Phys., 70 (1998) 223.

[2] Douglass J. K., Wilkens L., Pantazelou E. and Moss F., Nature, 365 (1993) 337.

[3] Levin J. E. and Miller J. P., Nature, 380 (1996) 165.

[4] Jaramillo F. and Wiesenfeld K., Nature Neurosci., 1 (1998) 384.

[5] Gluckman B. J., Netoff T. I., Neel E. J., Ditto W. L., Spano M. L. and Schiff S. J., Phys. Rev. Lett., 77 (1996) 4098.

[6] Stacey W. C. and Durand D. M., J. Neurophysiol., 83 (2000) 1394; 86 (2001) 1104.

[7] Rudolph M. and Destexhe A., J. Comput. Neurosci., 11 (2001) 19.

[8] DeFelipe J. and Farinas I., Prog. Neurobiol., 39 (1992) 563.

[9] Koch C., Biophysics of Computation (Oxford University Press) 1999, pp. 350-373.

[10] Brown D., Feng J. and Feerick S., Phys. Rev. Lett., 82 (1999) 4731.

[11] Feng J., Neural Networks, 14 (2001) 955.

[12] Hansel D., Mato G. and Meunier C., Europhys. Lett., 23 (1993) 367; Liu F., Hu B. and Wang W., Phys. Rev. E, 63 (2001) 031907.

[13] Feng J. and Zhang P., Phys. Rev. E, 63 (2001) 051902.

[14] Bair W., Koch C., Newsome W. and Britten K., J. Neurosci., 14 (1994) 2870.

[15] Salinas E. and Sejnowski T. J., J. Neurosci., 20 (2000) 6193.

[16] Burkitt A. N. and Clark G. M., Neural Comput., 13 (2001) 2639.

[17] Robinson H. P. C. and Harsch A., Phys. Rev. E, 66 (2002) 061902.

[18] Liu F., Wang J. F. and Wang W., Phys. Rev. E, 59 (1999) 3453; Yu Y., Wang W., Wang J. and Liu F., Phys. Rev. E, 63 (2001) 021907.

[19] Softky W. and Koch C., J. Neurosci., 13 (1993) 334.

[20] Aвbott L. F., Varela J. A., Sen K. and Nelson S. B., Science, 275 (1997) 220.

[21] Roskies A. (Editor), The binding problem, Neuron, 24 (1999) 7-125.

[22] Vertes R. P., Albo Z. and Di Prisco G. V., Neuroscience, 104 (2001) 619. 